\title{
Tagungsbericht zur Fachtagung „Praxisorientierte Psychotherapieforschung. Verfahrensübergreifende, patientenorientierte Aspekte und Kompetenzentwicklung“", 7./8. November 2014, Bundesministerium für Gesundheit, Wien
}

\author{
Michael Wieser · Brigitte Schigl · Henriette Löffler-Stastka \\ Eingegangen: 31. März 2015 / Angenommen: 12. April 2015 / Online publiziert: 20. Mai 2015 \\ (C) Springer-Verlag Wien 2015
}

Zusammenfassung Dieser Beitrag gibt einen Überblick über die wichtigsten Inhalte der Hauptvorträge bei der Tagung zur praxisorientierten Psychotherapieforschung, die am 7. und 8. November 2014 in Wien stattfand. Exemplarische Eindrücke aus den Workshops der Konferenz widerspiegeln einen Ausschnitt einer kreativen Arbeitsatmosphäre, offenen und anregenden Diskussion zu aktuellen Themen der Psychotherapieforschung, wie Wirksamkeits- und Versorgungsforschung, Prozessforschung, Ausbildungsforschung und Forschungsmethodik. Das längerfristige Ziel der stetigen Qualitätssteigerung und Kompetenzentwicklung in der Psychotherapieforschung und die Einbeziehung der Praktizierenden wurden durch die Tagung unterstrichen.

Schlüsselwörter Psychotherapieforschung · Qualitätssicherung $\cdot$ Kompetenzentwicklung

Diese Arbeit ist Teil des Leitthemas „Psychotherapieforschung“.

Aus dem Qualitätszirkel Psychotherapieforschung der Koordinationsstelle Psychotherapieforschung der Gesundheit Österreich $\mathrm{GmbH} /$ Geschäftsbereich ÖBIG.

\section{Wieser $(\square)$}

Institut für Psychologie, Alpen-Adria Universität Klagenfurt,

Universitätsstrasse 65-67,

9020 Klagenfurt, Österreich

E-Mail: Michael.wieser@aau.at

\section{B. Schigl}

Department für Psychotherapie und biopsychosoziale Gesundheit,

Donau Universität Krems,

Krems an der Donau, Österreich

H. Löffler-Stastka

Universitätslehrgang für Psychotherapieforschung, Klinik für

Psychoanalyse und Psychotherapie,

Medizinische Universität Wien,

Wien, Österreich
Conference on "Practice-based psychotherapy research". Overall aspects across therapeutic methods, patient-oriented aspects and development of competencies

Abstract An overview of all keynote presentations held at the conference on Practice-based Psychotherapy Research on Nov. 7th and 8th 2015 in Vienna is given. Exemplary impressions from the workshops of the conference reflect a portion of the creative working atmosphere, the open and stimulating discussion on current issues in psychotherapy research, such as efficacy and health services research, process research, research on training and competence development, and research methodology. The long-term goal of continuous quality improvement and development of competence in psychotherapy research as well as the involvement of practitioners was emphasized by the meeting.

Keywords Psychotherapy research · Quality assurance · Competence development - Patient- and practice-oriented research

\section{Eröffnung und Hauptreferate des ersten Tags}

Die Gesundheitsministerin Sabine Oberhauser eröffnet als erste Tagung nach ihrem Amtsantritt die zweite österreichische Psychotherapieforschungstagung, die von der neuen Koordinationsstelle Psychotherapieforschung in der Gesundheit Österreich GmbH (GÖG/ÖBIG) organisiert wurde. Sie bemerkte in dieser Eröffnung vor den 227 TeilnehmerInnen, dass PsychotherapeutInnen Wirksamkeitsbelege ihrer Behandlungen in Zahlen präsentieren können müssen - so wie andere Leistungserbringer im Gesundheitswesen. Michael Kierein, zuständiger Abteilungsleiter im Gesundheitsministerium (Sektion 2, Abteilung A/3), erinnert an den bisherigen Prozess der 
Qualitätsentwicklung im Bereich der Psychotherapie und nennt es als Ziel die bestmögliche Psychotherapie, wirksam und wirtschaftlich, für PatientInnen bereitzustellen.

Von Seiten des GÖG eröffnet und moderiert Gabriele Riess. Sie erklärt das Ziel dieser internationalen Tagung: Als nunmehr 2. Schritt nach der im November 2012 stattgefunden ersten Psychotherapieforschungstagung ginge es um das Sichtbarmachen der schon bestehenden österreichischen Forschungscommunity. Es sei sehr erfreulich, dass sich über 100 KollegInnen mit Abstracts auf den Call für die aktuelle Tagung gemeldet haben und diese weitgehend in den Panels berücksichtigt werden konnten. Die Tagung ist vom Qualitätszirkel Psychotherapieforschung inhaltlich geplant worden und konnte vom Team der Koordinationsstelle Psychotherapieforschung/GÖG (http://www.goeg.at/de/Bereich/Koordinationsstelle-Psychotherapieforschung.html) im Auftrag des Ministeriums organisiert werden.

Als erste Keynote erörtert Bernhard Strauß (Universitätsklinikum Jena) seine Sicht zur aktuellen Psychotherapieforschung unter dem Titel: Praxisorientierte Forschung zur Wirksamkeit von Psychotherapie - Zeichen eines Paradigmenwechsels. Er betont die hohe Effektstärke, die über alle Studien hinweg die Wirkung von Psychotherapie stabil belegt. Weiters, dass der Erfolg einer Psychotherapie nicht so sehr von dem Verfahren oder der Methode abhängig sei. Nötig wäre aber vermehrte Forschung im sogenannten „hard to reach“Bereich, sowie eine Erklärung für die Ergebnisvarianz der Wirkfaktoren. Auch konstatiert er, dass wir mehr Versorgungsforschung benötigen und eine höhere Aufmerksamkeit für Risiken von Psychotherapie. Die Zeiten der „Horse-race-studies“ seien jedoch vorbei, praxisorientierte Forschung zur Erlangung einer „practice based evidence" und patientenfokussierter Forschung das Gebot der Stunde. Er spricht sich für die Entwicklung transdiagnostischer Modelle psychischer Störungen aus und berichtet über das von ihm mitgeleitete QS-PSY Bay Projekt (vgl. http://dgpt.de/fileadmin/download/Mitteilungen/qs-psy-bay/Bernhard_Strauss.pdf).

Anton-Rupert Laireiter (Universität Salzburg) trägt in seinem Co-Referat „Wirksamkeit von Psychotherapie in der Praxis - Erkenntnisse aus einer missglückten Praxisstudie" mutig über die Gründe des Scheiterns einer geplanten Studie vor und entwickelt Ideen $\mathrm{zu}$ einem „Practice Research Network“. Es muss gelingen, genügend PraktikerInnen in die Forschung einzubinden, ansonsten kann die geforderte praxisnahe Forschung nicht stattfinden!

Ulrike Willutzki (Universität Witten-Herdecke) beschäftigt sich in der zweiten internationalen Keynote mit „Was macht eine/n gute/n TherapeutIn aus und lässt sich dafür etwas tun?" Sie fasst die Studien zu Innenerfolg zusammen, zu einem Drittel werden sehr gut wirksame, zu zwei Dritteln wirksame TherapeutInnen beschrieben, sowie in ca. $3 \%$ wird von TherapeutInnen berichtet, bei denen PatientInnen Verschlechterungen erleben. In kontrollierten Studien würden 5-10\% der Ergebnisvarianz durch TherapeutInnenfaktoren erklärt, in Versorgungsstudien sogar $17 \%$. Effektive TherapeutInnen haben weniger Schwankungen in ihren unterschiedlichen Behandlungen, und je schwerer beeinträchtigt die PatientInnen seien, umso eher wirke sich die Kompetenz der PsychotherapeutInnen aus. Sie entwirft weiters ein Bild eines/einer guten PsychotherapeutIn aus den Ergebnissen bisheriger Forschungsbemühungen und fordert als Schritte dorthin viel Supervision, Selbsterfahrung und Eigentherapie für PsychotherapeutInnen, sowie systematische Feedbackschleifen über den Therapieverlauf.

Svenja Taubner (Alpen-Adria-Universität Klagenfurt) befasst sich als Co-Referentin mit dem Thema „Kompetenzentwicklung von PsychotherapeutInnen in Ausbildung": Beziehungsfähigkeit sei die grundlegendste Therapeutenvariable und ein sicherer Bindungstyp befördere diese Selbstliebe als PsychotherapeutIn (Introjekt Affiliation). Diese bei AusbildungskandidatInnen zu untersuchen, ist ein Ziel des ehrgeizigen Projekts, das zurzeit an der Universität Kassel zur Ausbildung von PsychotherapeutInnen durchgeführt wird. Die zweite Fragestellung der Studie verknüpft Kompetenzentwicklung mit kritischen Lebensereignissen, Bindungsangst und Working Alliance.

Danach folgten am Nachmittag parallele Panels zu

- Wirkung und Wirksamkeit ambulanter Psychotherapie (A.L. Laireiter)

- Wirkung und Wirksamkeit stationärer Psychotherapie (M. Wieser)

- Rolle der Therapeuten - therapeutische Beziehung (G. Mehta)

- Ausbildung und Kompetenzentwicklung 1 (B. Schigl)

Exemplarisch werden im Folgenden einzelne Themen der Workshops herausgegriffen, um einige inhaltliche Foci genauer zu beleuchten.

\section{Session 2: Wirkung und Wirksamkeit stationärer Psychotherapie}

\section{Moderation: Michael Wieser}

Präsentiert wurden vier Vorträge und ein Poster aus dem Landesklinikum Mauer (Heigl-Steinhauser und Mayringer), der Reha-Klinik für seelische Gesundheit Klagenfurt (Senft), dem Otto-Wagner-Spital (Weipert) und dem Sozialmedizinischen Zentrum Baumgartner Höhe in Wien (Stastka). Als übergeordnete Frage ergab sich, ob die Kliniken mit ähnlichen PatientInnen sich nicht untereinander vergleichen könnten. Methodisch wurde quantitativ vorgegangen, zumeist im Sinne einer Evaluation. Eine Wartekontrollgruppe gab es nur in einem Falle, klinische Signifikanz wurde nachgefragt. Das praxisorientierte Vorgehen ist verfahrensübergreifend, weit über Psychotherapie hinaus gefasst. Gefragt nach dem Forschungseffekt für die Abteilung kam zur Antwort, dass Defizite differenziert angegeben wurden. Manche haben interne Qualitätssicherung, andere haben Kooperations- 
partnerInnen an Universitäten gefunden. Der Moderator schloss die Session mit dem Eindruck, dass durch den konstruktiven Austausch ein Motivationsklima zum Forschen entstanden ist.

Birgit Senft als Sprecherin der Session formuliert ihre Perspektive so:

\begin{abstract}
„Die Session fand in einer angenehmen Atmosphäre statt, in der die Vortragenden und Besucher reges Interesse sowohl an den jeweiligen therapeutischen Interventionen wie auch an den Ergebnissen zeigten. Allen präsentierenden Einrichtungen gemeinsam sind eine schwer belastete Klientel und die Erfahrung, dass die jeweiligen Behandlungsansätze hilfreich sind. Die Behandler Teams stellen sich kritische Fragen und wünschen sich mehr Prozessforschung, über die Qualitätssicherung und Evaluation hinausgehend. Bisher gibt es erst einzelne Ansätze zur Prozessforschung. Diskutiert wurden auch Möglichkeiten objektive Erfolgsparameter wie z. B. physiologische oder ökonomische Daten in die Bewertung des Therapieerfolgs einzubeziehen."
\end{abstract}

Im Plenum wurden sodann die einzelnen SessionBerichte von einem "speaker" und der ModeratorIn zusammengeführt. Anschließend gab es ein Treffen der wissenschaftlich Beauftragten aller fachspezifischen Ausbildungseinrichtungen. Im Foyer war ein Büchertisch aufgebaut. Der Abend schloss mit einem wohlschmeckenden Catering.

Am zweiten Tag begann die Tagung mit einer weiteren Key-Note:

John McLeod (Universität Oslo) sprach über die Nutzbarmachung und Bedeutung von Einzelfallstudien (Case Studies). Gute Case Studies fangen die Komplexität von Prozessen ein. Sie können nach verschiedenen Gesichtspunkten wie Narrative, Programmatische und Ergebnisorientierte Einzelfallstudien unterschieden werden und könnten nach Einhaltung von spezifischen Qualitätskriterien wie angereicherter Daten, Teamarbeit und Kritik nützlich für die Praxisschulung und Theorieentwicklung, sowie Konzeptforschung sein (siehe Beitrag in diesem Heft). Er betonte aber auch, dass dabei wie in einer Gerichtsverhandlung eine skeptische Perspektive eingenommen werden muss. Schließlich endet er mit Hinweisen über Websites mit Case-Study Material: dieses singlecasearchive.com/ bietet psychodynamisch Interessierten Ausgangsmaterial. Innerhalb von www. psychotherapyresearch.org wird eine Interest Section zum Thema Fallstudien eingerichtet.

Silke Gahleitner (Donau-Universität Krems) plädierte in ihrem Co-Referat über „Patient driven Psychotherapy - Die Perspektive der PatientInnen als vernachlässigte Dimension in Forschung und Praxis“. Sie konzentrierte sich in auf die Perspektive der PatientInnen und schlägt einen „mixed methods“ Ansatz vor, um in Studien zu triangulieren und unterschiedliche Perspektiven einzubringen. Dies führt sie am Beispiel der Studie „Risiken,
Neben-Wirkungen und Schäden von Psychotherapie, RISK" aus: Eine rechtzeitige Rückmeldung von Betroffenen und die Berücksichtigung deren Feedbacks durch die Innen könne unerwünschte Wirkungen bzw. Therapie-Fehler vermeiden oder korrigieren. Sie leitet aus diesen Überlegungen ein zukünftiges Projekt zur genauen Analyse der therapeutischen Beziehung im Prozess (aus PatientInnen wie TherapeutInnen-Sicht) ab.

Daran schlossen sich vier weitere Panels mit den Ergebnissen österreichischer Psychotherapieforschung an:

- Therapeutische Prozesse (M. Wieser)

- Spezielle Settings und Patientengruppen (C. Korunka)

- Forschungsleitende Diskurse in der Psychotherapie (B. Schigl)

- Ausbildung und Kompetenzentwicklung 2 (H. LöfflerStastka)

\section{Session 5: Therapeutische Prozesse}

\section{Moderation: Michael Wieser}

Die drei Vorträge und ein Poster hatten unterschiedliche Themen wie "Intersessionprozesse“ (Laireiter et al.), Wendepunkte (Kronberger), Prädiktoren (Hofinger) und Körperwahrnehmung (Grillmeier-Reder). Forschungsmethodisch waren von Zeitreihenanalysen bis zum Einzelfall zu finden. Gemeinsam war, dass keine Kontrollgruppen eingesetzt wurden. Die Beiträge waren praxisorientiert und zumeist verfahrensübergreifend. Machbar war dies durch Kooperationen mit einzelnen Universitäten und Diplomarbeiten.

\section{Session 4 und 8: Ausbildung und Kompetenzentwicklung $1+2$}

\section{Moderation: Brigitte Schigl und Henriette Löffler-Stastka}

Die Beiträge beschäftigten sich mit unterschiedlichen Elementen, die im Rahmen der Psychotherapieausbildung eine Rolle spielen. Ein Schwerpunkt lag auf der Selbsterfahrung, beispielsweise wurden eine qualitative Erhebung von Experteninterviews zum Thema Selbsterfahrung, sowie eine quantitativ-qualitative Studie zu diesem Thema vorgestellt. Im Sinne der Förderung einer breiten Diskussion der Forschungsmethodologie konnten methodische Fragen ausführlich erörtert und kritisch diskutiert werden. Zudem stand die Kompetenzentwicklung im Fokus, wie auch die Frage, wie Lebenserfahrung oder Reife operationalisierbar wären, oder wie diese Parameter mit interpersonellem Funktionieren und der therapeutischen Beziehung in Zusammenhang stehen können. Zur Kompetenzentwicklung und Ausbildungsforschung wurde zur Vernetzung weiters auf die „interest section“ der „Society of Psychotherapy Research“ hinge- 
wiesen (SPR Interest Section on Therapist Training and Development - SPRISTAD http://www.psychotherapyresearch.org/?page=SPRISTAD)

Über diese Konferenz wie über die erste von der GÖG organisierte Tagung im November 2012 (http://www. goeg.at/cxdata/media/download/berichte/psychotherapieforschung_wissenschaftliche_beratung_und_vernetzung.pdf) wird ein frei zugänglicher Tagungsbericht mit den Abstracts der Panelbeiträge und ausführlicheren Darstellungen der Hauptreferate ausgearbeitet (Im Moment unter http://www.goeg.at/de/GOEG-Aktuelles/ Praxisorientierte-Psychotherapieforschung207.html).

Insgesamt eröffnete die Tagung eine erfreuliche Perspektive auf die vielen schon existierenden Forschungsvorhaben der Universitäten und Ausbildungsvereine. Die Forschungsbeauftragten der Psychotherapie-Ausbil- dungseinrichtungen konnten sich miteinander vernetzen, all dies bei inhaltlich gut aufeinander abgestimmten und hochinteressanten Forschungsfragen. Es wird eine der Herausforderungen weiterer Forschungsbemühungen sein, die unterschiedlichen Projekte weiterzuentwickeln und zusammenzuführen - PraktikerInnen für Forschung zu gewinnen und fachspezifisch übergreifende Projekt-Designs anzudenken. Die Koordinationsstelle Psychotherapieforschung wird hier mit Hilfe des Qualitätszirkels Psychotherapieforschung Unterstützung bereitstellen.

Therefore, mind the gap! between science and practice, but do not underestimate the integrative function of Practice Research Networks! 